\title{
Electron Beam Induced Structural Modification of the Oxidized Silicon Micro-Clusters in ZnO Matrix
}

\author{
Umadapa Pal $\left({ }^{1}\right)$, Naoto Koshizaki $\left({ }^{2}\right)$, Shin-ya Terauchi $\left({ }^{2}\right)$ \\ and Takeshi Sasaki $\left({ }^{2}\right)$ \\ ( $\left.{ }^{1}\right)$ Instituto de Física, Universidad Autónoma de Puebla, Apdo. Postal J-48, Puebla, \\ Pue. 72570, Mexíco \\ $\left({ }^{2}\right)$ National Institute of Materials and Chemical Research (NIMC), AIST, MITI, 1-1 Higashi, \\ Tsukuba, Ibaraki 305, Japan
}

(Received August 20, 1997; accepted March 13, 1998)

PACS.61.80.Fe - Electrons and position radiation effects
PACS.61.46.+w - Clusters, nanoparticles, and nanocrystalline materials
PACS.61.82.Rx - Nanocrystalline materials

\begin{abstract}
Si} / \mathrm{ZnO}$ nano- and micro-composites were grown on $\mathrm{SiO}_{2}$ glass substrates by co-sputtering technique. The content of $\mathrm{Si}$ in the films was controlled by the no. of Si pieces placed on the $\mathrm{ZnO}$ target. The crystallinity of the composite films decreased on increasing the $\mathrm{Si}$ content in them and increased on post deposition thermal annealing. The dispersed $\mathrm{Si}$ in the $\mathrm{ZnO}$ matrix remained in the form of nano-particles with an average size value of $3.7 \mathrm{~nm}$ and does not depend on the fractional Si content in them. On thermal annealing, the size of the nano-particles did not change noticeably up to $600{ }^{\circ} \mathrm{C}$. For the thermal annealing at and above $700{ }^{\circ} \mathrm{C}$, the nano-particles aggregated to form micro-clusters with an average size value of $34 \mathrm{~nm}$. The micro-clusters were seen to be crystallized with distinct line structures in the TEM images and spots in the TED patterns. On high energy electron irradiation, the micro-clusters broke to form nano-particles of similar size as they were before the formation of micro-clusters.
\end{abstract}

\section{Introduction}

In recent years, the preparation and characterization of low dimensional composites made of group-IV elements, in particular, have attracted much interest due to their strong photoluminescence (PL) in the visible or near infrared (IR) region [1-5]. Though the preparation and behaviours of nano- and micro-crystals of $\mathrm{Si}$ and Ge have been reported by several workers [6-11], in most of the studies, $\mathrm{SiO}_{2}$ has been used as the matrix material for those optical functional composites. On the other hand, use of functional matrix materials like $\mathrm{TiO}_{2}, \mathrm{ZnO}$, $\mathrm{MgO}$ is rather very recent [12-15] and the influence of such photo-active surrounding hosts on the guest-host systems is not yet well understood. Use of such photo-active matrices for the preparation of low dimensional composites might have some special advantages.

In the present work, we tried to prepare $\mathrm{Si} / \mathrm{ZnO}$ nano-composites by co-sputtering technique. To the authors' knowledge, there is no previous report on the preparation or characterization of such composite systems. The composite films were deposited on $\mathrm{SiO}_{2}$ glass substrates followed 


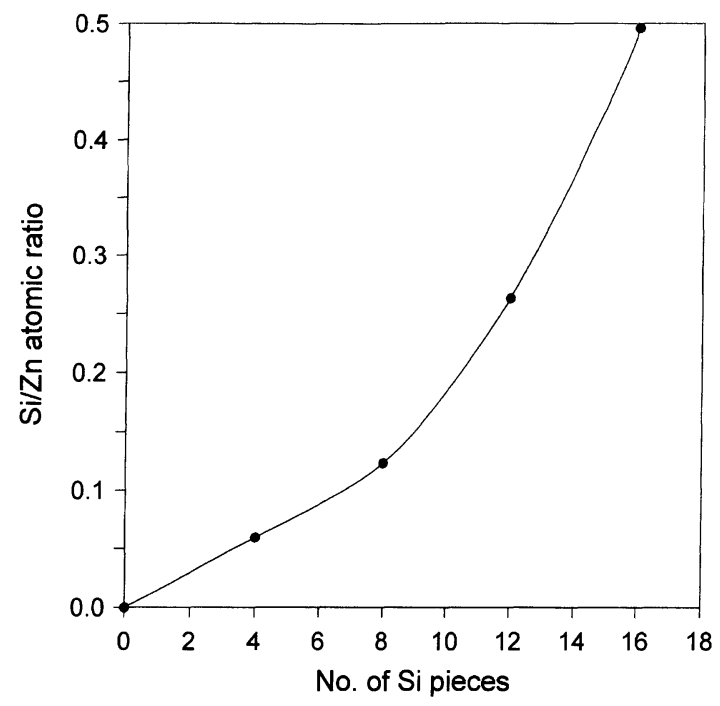

Fig. 1. - Variation of $\mathrm{Si} / \mathrm{Zn}$ atomic ratio in $\mathrm{Si} / \mathrm{ZnO}$ composite films with the no. of Si pieces placed on the $\mathrm{ZnO}$ target.

by a post deposition annealing at different temperatures in vacuum. The composition and the chemical states of the elements in the composites were studied by X-ray Photoelectron Spectroscopy (XPS) technique. Formation of Si nano-particles and their evolution on the increase of annealing temperature is studied by Transmission Electron Microscopy (TEM) technique. Formation of Si micro-clusters on thermal annealing beyond $700{ }^{\circ} \mathrm{C}$ and their subsequent changes on high energy electron irradiation are studied in details using TEM and Transmission Electron Diffraction (TED) techniques and presented.

\section{Experiments}

$\mathrm{Si} / \mathrm{ZnO}$ composite films were deposited on $\mathrm{SiO}_{2}$ glass substrates (Nihon Rika Garasu Kogyo) by co-sputtering method using an r.f. sputtering apparatus (Shimadzu HSR-521). Si wafers of $5 \mathrm{~mm} \times 5 \mathrm{~mm} \times 0.3 \mathrm{~mm}$ size were placed symmetrically on a $100 \mathrm{~mm}$ diameter ZnO target and sputtered with $100 \mathrm{~W}$ r.f. power at 10 mTorr Ar gas pressure. The content of Si in the films varied by changing the number of $\mathrm{Si}$ pieces on the $\mathrm{ZnO}$ target keeping the time of sputtering fixed (60 $\mathrm{min}$ ). The thickness of the films varied from $713 \mathrm{~nm}$ to $890 \mathrm{~nm}$ for the variation of Si pieces from 4 to 16 on the target. The as-deposited films with different Si content were annealed at $400{ }^{\circ} \mathrm{C}, 600{ }^{\circ} \mathrm{C}, 700{ }^{\circ} \mathrm{C}$ and $800{ }^{\circ} \mathrm{C}$ temperatures for $5 \mathrm{~h}$ in vacuum $\left(2 \times 10^{-6}\right.$ Torr $)$. The crystallinity of the as-deposited and annealed films were examined by X-Ray Diffraction (XRD) analysis using $\mathrm{CuK}_{\alpha}$ radiation (Rigaku, RAD-C). The chemical composition of the films and the chemical states of the individual elements were studied by a Perkin-Elmer (PHI 5600ci) X-ray Photoelectron Spectroscopy (XPS) system. For the TEM and TED observations, the composite films (about $25 \mathrm{~nm}$ thickness) with different Si content were deposited on carbon coated $\mathrm{NaCl}$ substrates. The films after transferring to the copper grids were annealed at different temperatures $\left(400-800{ }^{\circ} \mathrm{C}\right)$ in Ar atmosphere. A JEOL, JEM2000-FXII electron microscope was used for the TEM and TED observations on the films. 


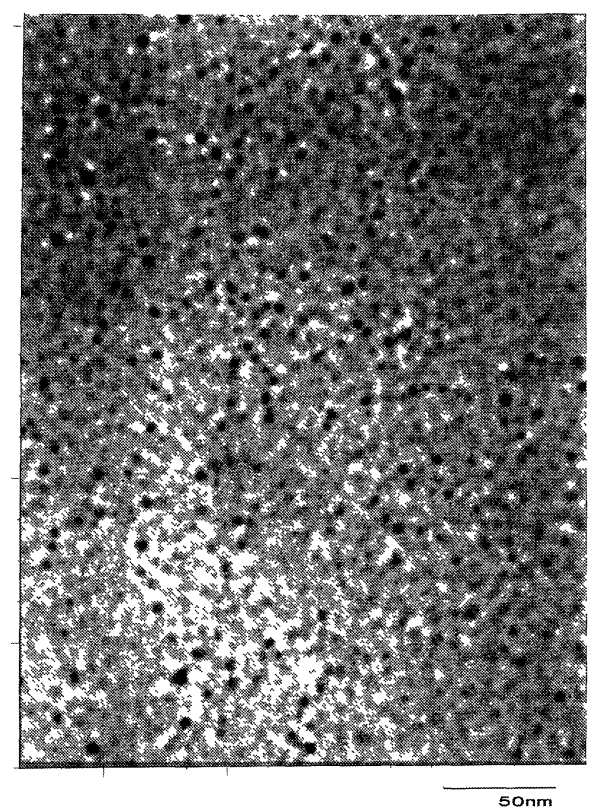

Fig. 2. - A typical TEM micrograph of as-deposited $\mathrm{Si} / \mathrm{ZnO}$ composite film prepared with 12 pieces of $\mathrm{Si}$ on the $\mathrm{ZnO}$ target.

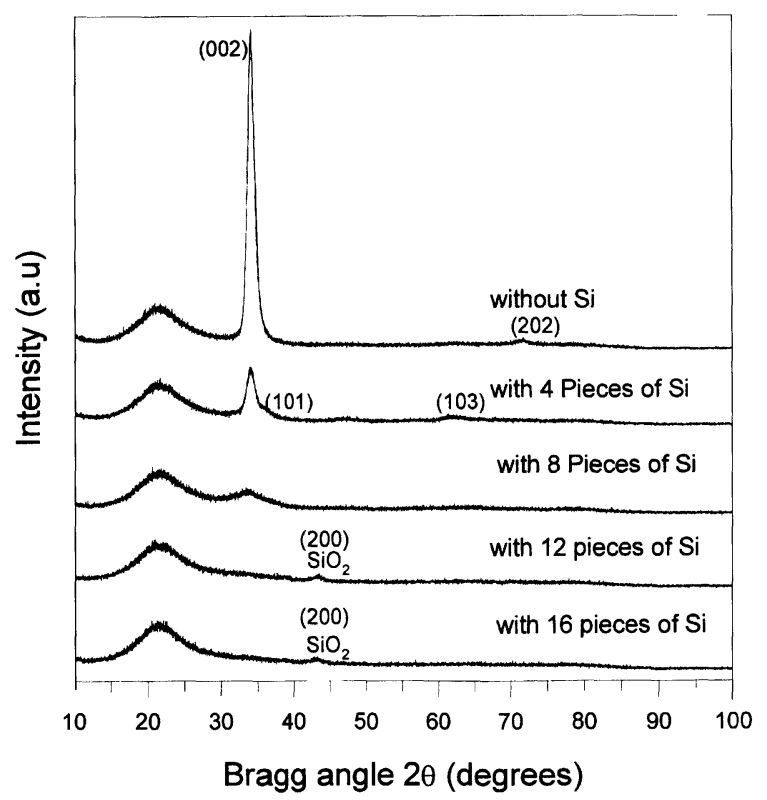

Fig. 3. - XRD patterns of as-grown $\mathrm{Si} / \mathrm{ZnO}$ films with different $\mathrm{Si}$ contents. The peak at about $22^{\circ}$ is due to the $\mathrm{SiO}_{2}$ substrate. 


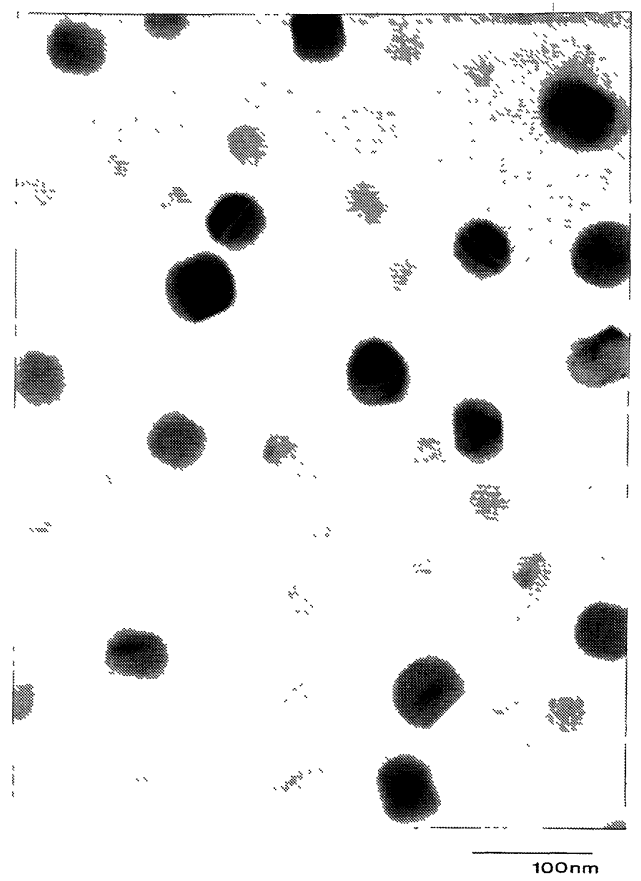

Fig. 4. - TEM micrograph of $\mathrm{Si} / \mathrm{ZnO}$ composite film after annealing at $800{ }^{\circ} \mathrm{C}$ for $5 \mathrm{~h}$.

Table I. - Interplanar spacings for SiO micro-clusters calculated from TED pattern.

\begin{tabular}{cc}
\hline Calculated $d$ values $(\AA)$ & Standard* $d$ values $(\AA)$ \\
\hline 1.930 & 1.930 \\
1.058 & 1.050 \\
0.966 & 0.960 \\
0.926 & 0.920 \\
0.855 & 0.860 \\
0.780 & 0.790 \\
0.741 & - \\
0.717 & - \\
0.674 & - \\
0.654 & - \\
0.630 & - \\
0.570 & - \\
0.555 & - \\
0.542 & - \\
0.535 & - \\
0.529 & - \\
0.517 & - \\
0.490 & - \\
0.483 & - \\
\hline
\end{tabular}

* Kurdymov et al., Inorg. Mat. 2 (1966) 1539, (JCPD-ICDD 30-1127). 


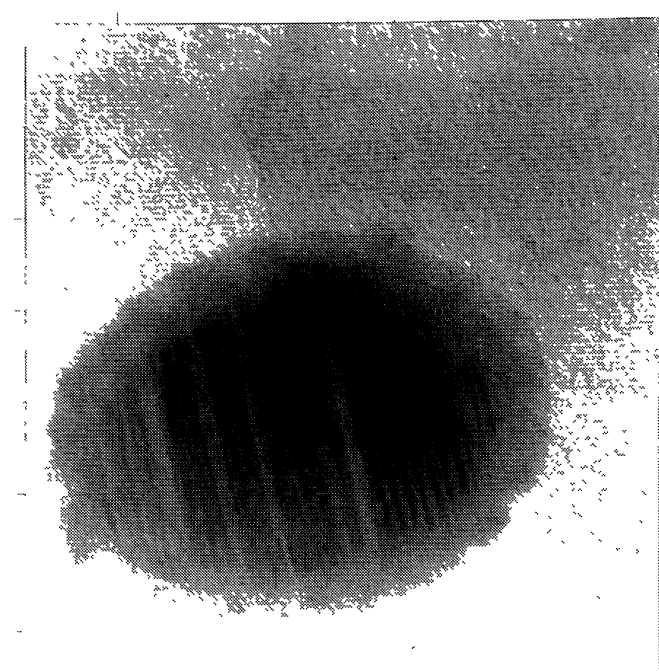

a

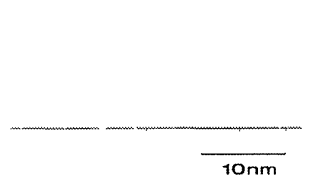

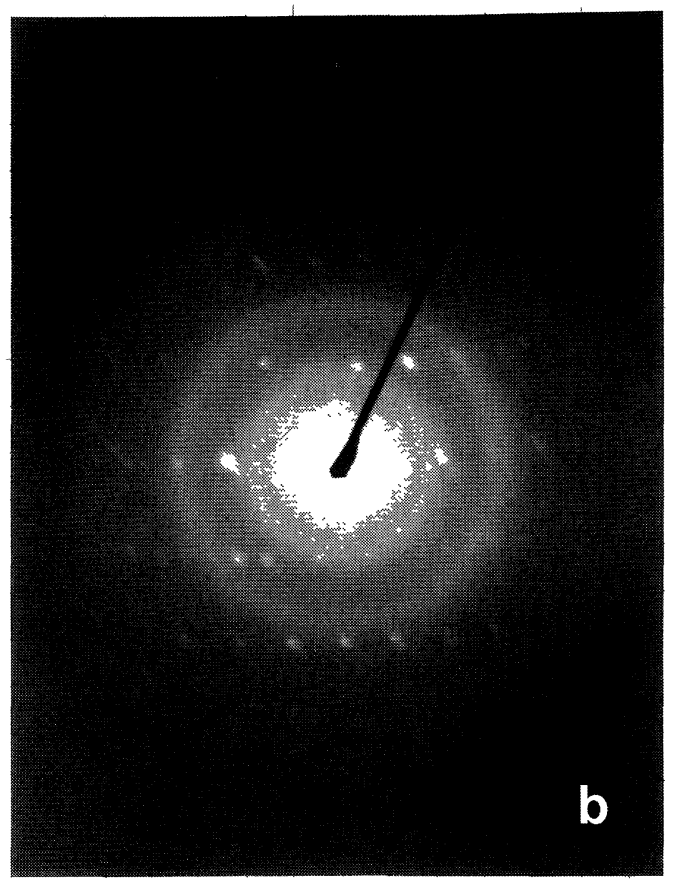

Fig. 5. - (a) TEM photograph of a typical crystalline micro-cluster formed on annealing at $800{ }^{\circ} \mathrm{C}$ for 5 h. (b) TED pattern of the crystallized micro-cluster shown in Figure 5a.

\section{Results and Discussion}

Figure 1 shows the $\mathrm{Si} / \mathrm{Zn}$ atomic ratio in the as-deposited composite films as a function of $\mathrm{Si}$ pieces placed on the $\mathrm{ZnO}$ target. The $\mathrm{Si} / \mathrm{Zn}$ atomic ratio was calculated from $\mathrm{Si}_{2} \mathrm{p}_{3 / 2}$ and $\mathrm{Zn} 2 \mathrm{p}_{3 / 2}$ peak areas in XPS spectra of the films. The $\mathrm{Si} / \mathrm{Zn}$ atomic ratio in the films increased with the increase of Si pieces and could easily be controlled by changing the number of Si pieces on the $\mathrm{ZnO}$ target.

Figure 2 shows a typical TEM micrograph of as-deposited $\mathrm{Si} / \mathrm{ZnO}$ composite film prepared with 12 pieces of $\mathrm{Si}$ wafers (corresponds to $\mathrm{Si} / \mathrm{Zn}=0.265$ ). We can observe the nanoparticles of size ranging from 2 to $4 \mathrm{~nm}$ dispersed in the matrix homogeneously. From the XRD patterns of the films it is observed that the $\mathrm{ZnO}$ films with no $\mathrm{Si}$ or with lower Si content are of polycrystalline in nature. With the increase of Si content, the crystallinity of the composite films decreases. In fact, the films prepared with 12 pieces of $\mathrm{Si}$ or more were amorphous. In Figure 3, the XRD spectra of the as-grown films with different Si content are presented. With the increase of annealing temperature, though the crystallinity of the films increased, the dimension of the nanoparticles did not increase noticeably. On annealing the films (irrespective of the amount of $\mathrm{Si}$ content in them) at $700{ }^{\circ} \mathrm{C}$ or higher temperatures, the nanoparticles aggregated to form micro-clusters. In Figure 4, a typical TEM picture of the micro-clusters formed after annealing at $800{ }^{\circ} \mathrm{C}$ is shown. The average size of micro-clusters was $34 \mathrm{~nm}$ (dispersive, size value varied from $10 \mathrm{~nm}$ to $60 \mathrm{~nm}$ ). The average size of the clusters did not depend on the Si content in the films. However, the density of the micro-clusters was higher 


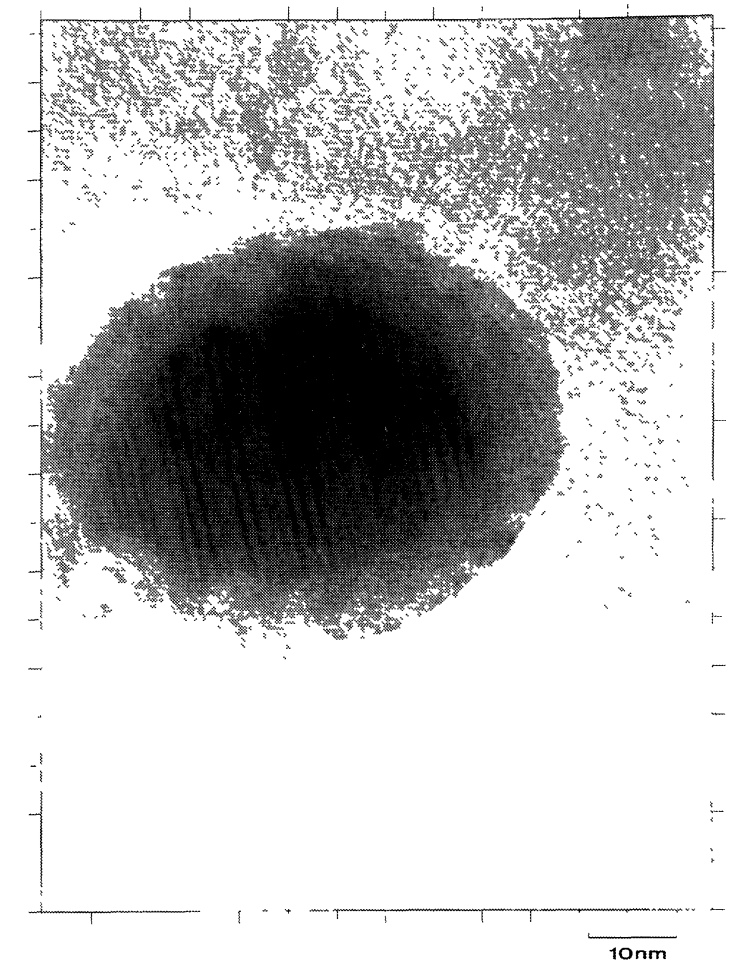

Fig. 6. - TEM photograph of the crystallized micro-cluster shown in Figure 5a after 2 min electron $(200 \mathrm{keV})$ irradiation. A well crystallized nano-particle after its break-off from the micro-cluster is marked by a in Figure 5a.

for the films with higher Si content. From the TEM observation of the micro-clusters, it is seen that, most of them are crystallized. In Figures 5a and b, typical TEM image of a crystallized micro-cluster and its TED pattern are shown respectively. The micrograph (Fig. 5a) shows a crystal structure including twin lamellae and the diffraction pattern (Fig. 5b) exhibits a singlecrystal lattice in zone-axis orientation and a secondary lattice, probably in twin orientation. The angle between the lattice rows and the paired spots in the TED pattern indicate that the micro-clusters are not perfect single crystals. Therefore we can designate the clusters as bi-crystals or combination of more than one crystals with slightly different orientations. The calculated $d$ values from the diffraction spots agreed closely with the reported $d$ values of $\mathrm{SiO}$ (Tab. I). Due to unavailability of a sufficient number of $d$ values and their corresponding indices in literature at present, we could not calculate the zone-axis orientations of the lattice planes. However, from the angular deviation of the rows of the diffraction spots we could estimate at about $4.4^{\circ}$ the angle between the two zone-axes of the two sets of lattice planes in the sample. From the XPS study of the Si2p emission peak position, we could see that in none of the films the dispersed $\mathrm{Si}$ was in its elemental state. The peak position of $\mathrm{Si}$ in $\mathrm{Si} / \mathrm{ZnO}$ composites (varied from 101.6 to $102.8 \mathrm{eV}$ ) remained in between the peak positions of elemental $\mathrm{Si}(99.2 \mathrm{eV})$ and $\mathrm{SiO}_{2}(103.9 \mathrm{eV})$. So, in the micro-clusters, Si remained in the $\mathrm{SiO}_{X}$ $(0<X<2)$ chemical state.

On irradiation of high energy $(200 \mathrm{keV}$ in the present case) electrons to the micro-clusters, the crystallinity breaks from the border to the centre with time. The state of a crystallized micro-cluster after 2 min electron irradiation is shown in Figure 6. The crystallized 


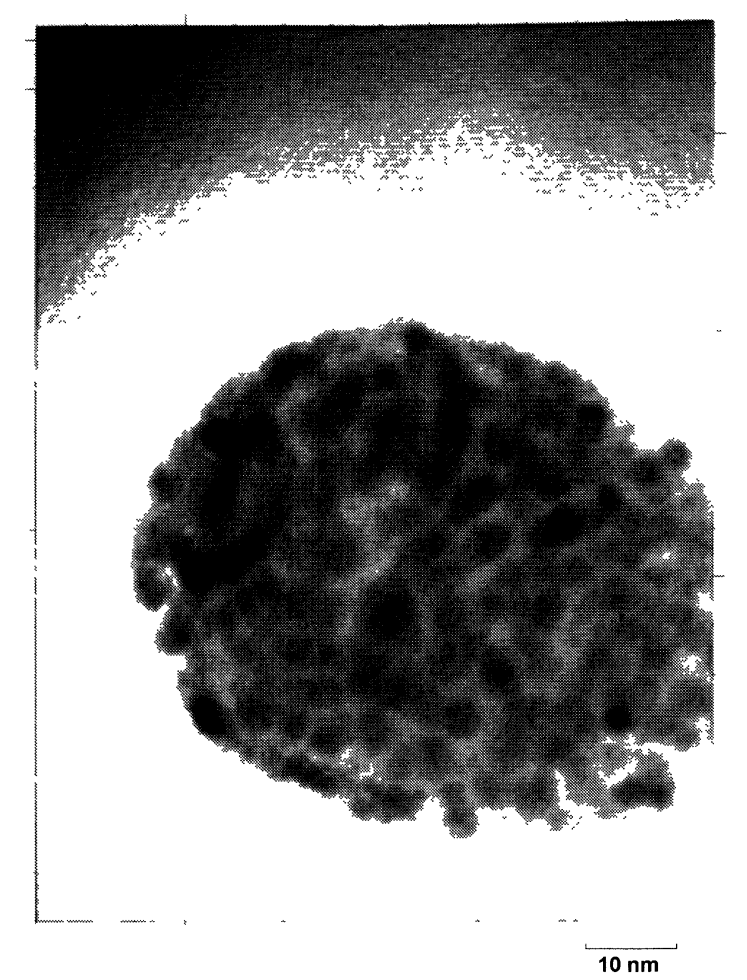

Fig. 7. - A completely broken micro-cluster after electron $(200 \mathrm{keV})$ irradiation for about 8 minutes.

micro-cluster broke to form crystallized nano-particles. Such a crystallized nano-particle after its break off from the micro-cluster can be seen in Figure 5a (marked as a). After an irradiation of high energy electrons for sufficient time, the whole micro-cluster breaks to closely spaced nano-particles. In Figure 7, the state of a completely broken micro-cluster after about 8 min electron irradiation is shown. In Figure 8, the TED pattern of such a broken cluster is presented. The unoriented diffraction spots in the TED pattern illustrate that, after complete transformation of the crystallized micro-cluster to closely spaced nano-crystals, the crystallinity of the cluster, as a whole, vanishes but each of the nano-particles remains crystallized with an arbitrary orientation among themselves. On electron irradiation, the crystallized microclusters break and a shell around the crystal extending towards its centre is formed. Though this thin outer shell around the crystal seems to be amorphous in nature, some dark contrast inside each of the grains of the shell can be observed and the TED patterns of completely broken micro-clusters do not correspond to a amorphous structure. A structural transformation of quasi-crystalline $\mathrm{Al}_{62} \mathrm{Cu}_{20} \mathrm{Co}_{15} \mathrm{Si}_{3}$ alloy under high energy electron irradiation has been reported by Gasa et al. [16]. The state of the quasi-crystalline alloy after electron irradiation was amorphous in their samples. However, in our case, the crystalline micro-clusters broke to form crystalline nano-particles. As the dimension of the nano-particles in our samples was smaller than the probing electron beam spot and as their orientations were arbitrary, we could not detect the orientation or crystalline phase of individual nano-particles. 


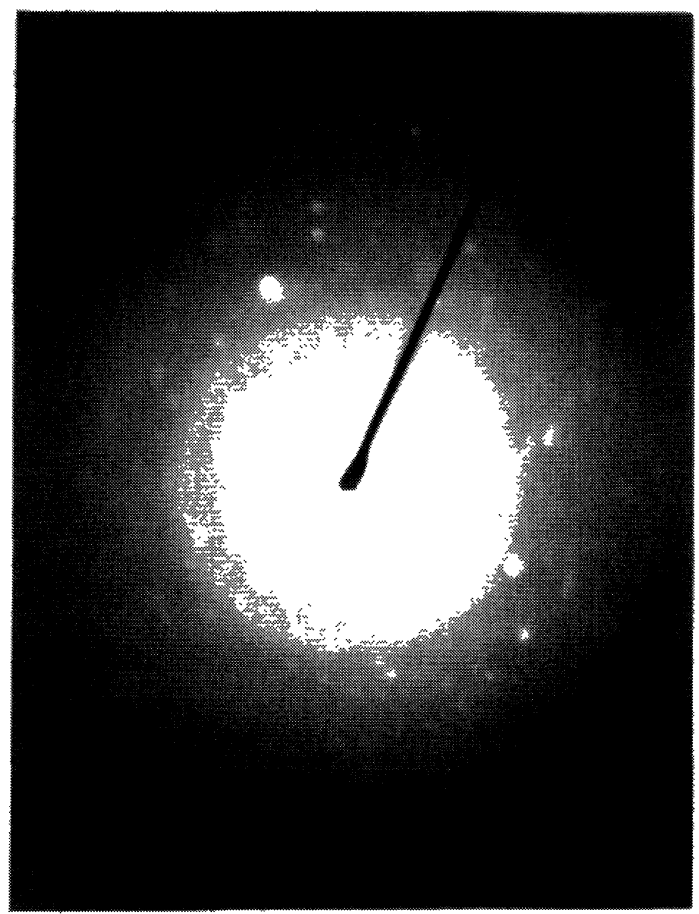

Fig. 8. - TED pattern of the broken micro-cluster shown in Figure 6.

\section{Conclusions}

We could prepare oxidized Si nanoparticles and micro-clusters in $\mathrm{ZnO}$ matrix for the first time by co-sputtering technique. The Si content in the films initially increased linearly and then almost exponentially on the no. of Si pieces on the $\mathrm{ZnO}$ target. The dispersed $\mathrm{Si}$ in all the as-deposited composite films formed nanoparticles with average size value $3.7 \mathrm{~nm}$ and the value did not depend on the content of $\mathrm{Si}$ in the films. The chemical state of $\mathrm{Si}$ in the composite films was $\mathrm{SiO}_{X}(0<X<2)$ even after thermal annealing. On annealing of the composite films at and above $700{ }^{\circ} \mathrm{C}$, the mano-particles aggregated to form crystallized micro-clusters which were unstable to high energy electron irradiation.

\section{Acknowledgments}

U. Pal thanks JISTEC, Japan for the STA award. The work is partially supported by CONACyT grant (No. 1351-PA).

\section{References}

[1] Hayashi S., Kataoka M. and Yamamoto K., Jpn J. Appl. Phys. 32 (1993) 274.

[2] Yoshida S., Handa T., Tanabe S. and Soga N., Jpn J. Appl. Phys. 35 (1996) 2694. 
[3] Maeda Y., Tsukamoto N., Yazawa Y., Kanemitsu Y. and Masumoto Y., Appl. Phys. Lett. 59 (1991) 3168.

[4] Kanzawa Y., Kageyama T., Takeoka S., Fujii M., Hayashi S. and Yamamoto K., Solid State Commun. 102 (1997) 533.

[5] Hayashi S., Nagareda T., Kanzawa Y. and Yamamoto K., Jpn J. Appl. Phys. 32 (1993) 3840.

[6] Takagi H., Ogawa H., Yamazaki Y., Ishizaki A. and Nakagiri T., Appl. Phys. Lett. 56 (1990) 2379.

[7] Littau K.A., Szajowski P.J., Muller A.J., Kortan A.R. and Brus L.E., J. Phys. Chem. 97 (1993) 1224.

[8] Kanzawa Y., Hayashi S. and Yamamoto K., J. Phys.: Condens. Matter 8 (1996) 4823.

[9] Fujii M., Inoue Y., Hayashi S. and Yamamoto K., Appl. Phys. Lett. 68 (1996) 3749.

[10] Vial J.-C., Canham L.T. and Lang W., in Light emission from Silicon (North Holland, Amsterdam, 1994).

[11] Morisaki H., Ping F.W., Ono H. and Yazawa K., J. Appl. Phys. 70 (1991) 1869.

[12] Sasaki T., Rozbicki R., Matsumoto Y., Koshizaki N., Terauchi S. and Umehara H., Mat. Res. Soc. Symp. Proc. 457 (1997) 425.

[13] Koshizaki N., Yasumoto K., Terauchi S., Umehara H., Sasaki T. and Oyama T., Nanostruct. Mat. 9 (1997) 587.

[14] Lee M.H., Chang I.T.H., Dobson P.J. and Cantor B., Mater. Sci. Eng. A179/A180 (1994) 545.

[15] Zhao G., Kozuka H. and Yoko T., Thin Solid Films 227 (1996) 147.

[16] Gasa J.R., García R. and Yacamán M.J., Rad. Phys. Chem. 45 (1995) 283. 